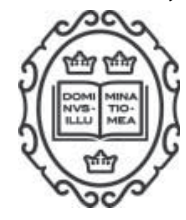

\title{
A Vindication of Logicism ${ }^{\dagger}$
}

\author{
Peter Roeper \\ School of Philosophy, Australian National University, Canberra, ACT 0200, Australia \\ E-mail: peter.roeper@anu.edu.au
}

\begin{abstract}
Frege regarded Hume's Principle as insufficient for a logicist account of arithmetic, as it does not identify the numbers; it does not tell us which objects the numbers are. His solution, generally regarded as a failure, was to propose certain sets as the referents of numerical terms. I suggest instead that numbers are properties of pluralities, where these properties are treated as objects. Given this identification, the truth-conditions of the statements of arithmetic can be obtained from logical principles with the help of definitions, just as the logicist thesis maintains.
\end{abstract}

\section{LOGICISM}

Logicism, as Frege conceived it, contends that the truths of arithmetic are logical truths; they can be derived by invoking only logical principles and definitions. Arithmetic is here understood, in the first instance, as the theory of natural numbers. Frege's aim was to show that the true statements of arithmetic can be justified in terms of principles of logic with the help of definitions of mathematical concepts in terms of logical ones.

Investigation of the use of number vocabulary in pre-mathematical language prompts Frege to identify equinumerosity, expressed by the relation 'there are as many ... as there are ...', as the logical notion that underlies the concepts of arithmetic. The central principle connecting arithmetic with logic is Hume's Principle:

$$
\text { the number of } F \mathrm{~s}=\text { the number of } G \mathrm{~s}
$$

there are as many $F$ s as $G$ s.

\footnotetext{
${ }^{\dagger}$ I am grateful to David Bostock and to the referees for critical and most helpful comments.
}

Philosophia Mathematica (III) Vol. 00 No. 0 (C) The Author [2015]. Published by Oxford University Press. All rights reserved. For permissions, please e-mail: journals.permissions@oup.com 
This principle is, both for Frege and for later defenders of logicism, the starting point for the logicist account of arithmetic.

Before turning to the task of defending logicism it is necessary to clarify the grammar of Hume's Principle and of attributions of number. The natural understanding of equinumerosity is that certain objects, the $F$ s, stand in this relation to certain, perhaps different, objects, the Gs. Similarly, an ordinary number statement like 'there are nine planets' appears to be attributing a number to certain objects, namely the planets. This is the view I will adopt. General nouns are taken to be referring expressions that stand for (pluralities of) objects, e.g., '(the) planets' refers to the planets, i.e., to each planet and to nothing else.

\subsection{Can Numbers Be Predicated of Parts of Reality?}

The view that in a typical number statement a number property is predicated of a plurality of objects is, or at least appears to be, in direct conflict with Frege's views. Frege holds that what a number predicate applies to cannot be a part of reality because without differentiation into units of counting no answer to the question 'how many are there?', posed in regard to that part of reality, can be given. A concept is needed which achieves the required differentiation; an ascription of number therefore contains a judgment about a concept. Concepts are expressed by predicates, and so Frege concludes that a number predicate is a second-level predicate attached to a first-level predicate. The number predicate expresses a concept under which first-level concepts fall.

The view that nouns are plural referring expressions also when they occur in number statements is unaffected by Frege's argument. A plural term, e.g., 'the planets', can be said to pick out a part of reality. But it does not do this in an undifferentiating way. The heavenly bodies that the term refers to are distinct from one another. Hence the statement 'the planets are nine' in which a number is predicated of them is perfectly significant. The concept of being a planet does indeed play a role in differentiating the referents of the plural term one from another. For Frege the concept planet is the reference of the predicate 'is a planet'. One can say that a concept is a property, but one must keep in mind that properties are understood predicatively and that the identity of a property goes with its extension.

The planets are just the objects that have the property of being a planet. Therefore this property is crucially involved in determining what the plural term 'the planets' refers to. One can say that the number statement 'the planets are nine' or 'there are nine planets' says something about the concept planet. But one does not have to say that the number predicate 'there are nine' is predicated of the concept. Frege's observation that a concept is needed in order to give us something in respect of which the question 'how many?' makes sense is perfectly compatible with the view taken here that the objects referred to by a plural term are (together) the bearers of number properties. 


\subsection{Hume's Principle and the Identity of Numbers}

Returning to Hume's Principle I say that the letters ' $F$ ' and ' $G$ ' are place-holders for plural terms. Using obvious shorthand Hume's Principle becomes

$$
n r(F)=n r(G) \equiv F \approx G .
$$

In mathematical practice numbers are treated as objects: there are singular terms that refer to individual numbers and plural terms that refer to pluralities of numbers, and numbers can constitute the range of quantifiers. Accordingly, the expression 'the number of $F \mathrm{~s}$ ' is what it appears to be, a singular term that refers to a number. It does not follow that natural numbers are objects in any metaphysically significant sense. It is sufficient that numbers can be presented or treated as objects. To treat an item as an object is simply to permit oneself to refer to it by means of a singular term. The only justification needed is that the use of such referring expressions is explained by specifying conditions of identity and satisfaction conditions of relevant predicates.

Hume's Principle appears to give us an insight into the nature of numbers. But it does not identify the numbers. As Frege recognised, Hume's Principle provides a criterion of identity for numbers; but adopting the principle as true does not succeed in defining the functor 'the number of', which is the one un-interpreted expression in the principle, and so the principle cannot tell us which objects are the natural numbers. Any progression can play the role of the numbers with the relationship $n$ is the number of $X s$ between numbers and pluralities appropriately adjusted so that Hume's Principle is satisfied.

Frege's Caesar problem illustrates the point. While the principle can tell us whether $m$ and $n$ are the same or different numbers, it does not help us answer the question whether the number 4 is or is not Julius Caesar, i.e., whether numbers are to be found among familiar entities, rather than being a quite different kind of object.

A plausible thought at this point is that the content of Hume's Principle has not been fully exploited. Numbers have properties that distinguish them from other types of object. A congruence property (under equinumerosity) is a property that applies to a plurality only if it applies to all equinumerous pluralities. E.g., '. . . can be evenly divided' is such a property. There is a corresponding property of numbers: $n$ has the property, namely being an even number, if, whenever it is true that $n$ is the number of $F$ s then the $F$ s can be evenly divided. In this fashion there is for every congruence property $\Phi$ a corresponding property of numbers $\varphi: \varphi(n) \equiv(\forall X)(n=n r(X) \supset \Phi(X))$. These properties $\varphi$ are the arithmetically relevant properties. Can we not distinguish the numbers from other types of objects as being those whose properties are the congruence properties under equinumerosity?

Although objects of already recognised kinds do not seem to have properties that involve equinumerosity this is not a sufficient reason for concluding that they cannot be numbers. As long as it is not a requirement to accept that the numbers have only those properties that are derived from congruence properties of pluralities, there is no compelling reason to reject the possibility that numbers 
can be identified with already recognised objects. Hume's Principle by itself does not rule out the possibility that Julius Caesar - as an element of some infinite progression - is the number 4 . One can understand this in the sense that Julius Caesar can play the role of 4, and it is in this role that Julius Caesar would acquire the relevant congruence properties. One must conclude with Frege that Hume's Principle cannot be regarded as a definition of the numbers.

\subsection{Frege's Program}

Since Hume's Principle alone does not specify what items the numbers are, it seems that an explicit identification of a category of objects is needed, a category for which it can be shown that Hume's Principle is satisfied. Frege chooses sets of a certain kind, either sets of sets or sets of concepts. E.g., the number 3 is the set of all 3-membered sets or the set of all concepts that have three objects falling under them. Given his identification Hume's Principle can be proved, and Frege proceeds to derive the Peano axioms.

Frege's logicist program has been deemed to be a failure. Two main reasons have been given for this assessment. I do not think that either of them is conclusive. The first criticism points to the inconsistency in Frege's logic due to the unrestricted set-abstraction principle (often referred to as Frege's Axiom $V$ ),

$$
\{x \mid F x\}=\{x \mid G x\} \equiv(\forall x)(F x \equiv G x) .
$$

The inconsistency is, however, hardly relevant, since Frege's proof of the Peano axioms does not essentially depend on his inconsistent principle of set identity [Heck, 1993]. In other words, the reasoning employed by Frege can be justified on the basis of standard axiomatic set theory.

The other main objection is that Axiom V is of the same form as Hume's Principle in having an identity on one side of the bi-conditional and an equivalence on the other. It is therefore just as much exposed to the Caesar problem and consequently cannot be used to solve that problem. ${ }^{1}$ There is, however, a significant difference. Frege's logic, like the standard first-order calculus, is extensional, which means that co-extensiveness of predicates is the first-order analogue of identity. Hence the set-forming functor is 1-1; it has an inverse. If $s$ is the set obtained from $F x$, i.e., $s=\{x \mid F x\}$, then the predicate $x \in s$ is co-extensive with $F x,(\forall x)(x \in s \equiv F x)$.

With identities on both sides of the bi-conditional it is possible to interpret set abstraction as a formal move. Although $\{x \mid F x\}$ and $F x$ belong to different grammatical categories, their semantic counterparts are the same. $\{x \mid F x\}$ is a singular term that is used to talk about the objects that satisfy the predicate $F x$ as if they were a single object. On this understanding the functor 'the set of ...' has a fixed interpretation; it is a logical constant. In Axiom $\mathrm{V}$ the functor is therefore not subject to alternative interpretations and the Caesar problem

\footnotetext{
${ }^{1}$ This point has been urged by Dummett [1998].
} 
does not arise. Axiom $\mathrm{V}$ is then not an (attempted) definition; rather it is a logical truth that records how properties and sets are related. I am not saying that Frege either would or could accept this interpretation, merely that it can be used to counter Dummett's criticism.

Neither of the two objections presents an unanswerable problem for the identification of numbers with sets. However, Frege's choice is unsatisfactory for other reasons. Firstly, the choice of sets is not well motivated. Frege offers no reason for the identification of numbers with sets other than that Hume's Principle can be proved if the identification is made. Secondly, taking sets as numbers introduces an undesirable element of contingency into arithmetic, since it is a factual matter which 3-membered sets there are and which concepts have three instances. The identification of numbers with sets therefore undermines the claim that numbers are logical objects. Thirdly, if numbers are taken to be sets of sets then circularity results. e.g., the set consisting of the numbers 2 , 3 , and 4 , i.e., the set $\{2,3,4\}$, is then a member of the number 3 . This means that 3 is a member of a member of itself and can therefore not be extensionally defined.

Finally, the assumption that numbers are sets of equinumerous sets prompts Frege to define individual numbers other than 0 as sets of sets that are equinumerous to a particular instance of the number in question. The number $n$ is the set of sets that are equinumerous to the set of numbers smaller than $n$. As a consequence there is, in his account, no connection between numbers and numerical quantifiers, between the number $n$ and $\left(\exists_{n} x\right)(\ldots x \ldots)$. In Frege's logical system simple attributions of number 'there are $n F$ s' are best construed as predicating a numerical quantifier of $F$ : ' $\left(\exists_{n} x\right) F x$ '. Instead, Frege has to adopt the not very plausible analysis 'the number of $F \mathrm{~s}=n$ '. So, while numerical quantifiers can easily be expressed in Frege's logical calculus, there is no straightforward explanation of how ' $\left(\exists_{9} x\right) x$ is a planet' can have the same content as 'the number of planets $=9$ '.

\subsection{Neo-Logicism}

Close study of Frege's reasoning in Grundgesetze der Arithmetic [1893/1961] has revealed that the identification of numbers with sets plays no role in the derivation of the Peano Axioms from Hume's Principle [Heck, 1993]. For Frege, identifying numbers as certain sets justifies Hume's Principle as a logical truth. No further use is made of the identification. Using second-order logic, Hume's Principle by itself is sufficient for the derivation of the basic principles of arithmetic.

This is the starting point of the neo-Fregean version of logicism. ${ }^{2}$ Hume's Principle by itself is claimed to be sufficient as a basis for the arithmetic of the natural numbers. The principle introduces the numbers by abstraction as a separate category of objects. This amounts to postulating that there is a

\footnotetext{
${ }^{2}$ The comprehensive neo-logicist text is [Hale and Wright, 2001].
} 
category of objects whose relevant properties are just the congruence properties under equinumerosity.

The main difficulties with the neo-logicist account are those that Frege himself points to. Given that the recourse to set theory is rejected, how can Hume's Principle be justified; how can it be shown to be a logical truth or to be analytic? Can the possibility of multiple interpretations of the functor 'the number of' (the Caesar problem) really be avoided? These are issues of continuing debate [Tennant, 2014]. To my mind neo-logicism has not succeeded in dissolving these difficulties. I believe that in order to judge that Hume's Principle is analytic it has to be settled first what kind of item the numbers are. ${ }^{3}$ But even if one accepts the neo-Fregean program, one can appreciate an approach that offers an explicit and clearly motivated identification of the numbers that neo-logicism postulates.

Having rejected both the identification of numbers with sets and the neoFregean reliance on Hume's Principle alone, we find ourselves at the same place as before: Hume's Principle needs to be supplemented with a definition of the numbers. I propose that the natural numbers are properties of pluralities. Though not implied by Hume's Principle, this is not an arbitrary identification. While it goes against some of Frege's views, I think the proposal can nevertheless be recognised as a version of logicism. Hume's Principle is the guide, so to speak, in developing the proposal.

\section{NUMBERS ARE PROPERTIES OF PLURALITIES}

\subsection{Equivalence Relations and Properties}

The question before us is: what kind of item are the numbers? Hume's Principle tells us that numbers are associated with pluralities in such a way that for a given plurality there is a unique number which is also associated with all equinumerous pluralities and no others. While the sets Frege takes to be numbers satisfy this condition, they are not a satisfactory choice, as I have argued, and there is no independent reason for making this choice.

Now the association of numbers with equinumerosity is not the only case in which an association exists between an equivalence relation and certain items. Frege himself provides the example of the relation between parallelism of lines and directions as being analogous to the relation between equinumerosity and numbers. Parallel lines have the same direction. But although Frege, of course, does not say this, directions are properties. The weather vane can have the North-South direction at one time and the West-East direction at another. Again, equi-ponderous material objects have the same weight and weights are properties. It is easy to extend the list of examples. In most, if not all, such cases items that stand in the equivalence relation have something in common, and what they have in common is a property. The following principle expresses

\footnotetext{
${ }^{3}$ For detailed criticism along these lines see [Trueman, 2014].
} 
the idea.

When an equivalence relation is defined over items of some kind, then there is a spectrum of properties such that two such items have the same property just when the equivalence relation holds between them.

Applying this principle in the present case we only need to take into account that the relata of the equivalence relation of equinumerosity are pluralities. Therefore the properties in question are properties of pluralities. And one is led to the suggestion that numbers are certain properties of pluralities, numerical properties. It remains to be determined which properties of pluralities they are.

There is, however, a problem: it has been accepted that numbers are objects. Hume's Principle itself refers to numbers by means of singular terms, i.e., it treats them as objects. So it needs to be clarified how numbers can be both objects and properties.

\subsection{Properties Treated as Objects}

We have arrived at the view that numbers are properties of pluralities and that numbers can be referred to by singular and plural terms. The tension between the two aspects of the suggestion can be resolved. For while the standard way of representing a property is to use a predicate, the familiar $\lambda$-notation allows one to introduce the property by means of a singular term. If $\Phi(X)$ is a predicate which expresses the property, then $\lambda_{X} \Phi(X)$ is a singular term introducing the property as an object. The identity conditions of properties as objects are familiar. Predicates $\Phi$ and $\Psi$ express the same property if they are necessarily co-extensive. Hence ${ }^{4}$

$$
\lambda_{X} \Phi(X)=\lambda_{X} \Psi(X) \equiv(\forall X) \square(\Phi(X) \equiv \Psi(X)) .
$$

The principle links two different ways of introducing a property: on the one hand there are predicates, understood intensionally, which express the property; on the other hand there are $\lambda$-terms that refer to the property. The move from predicate to singular term can be called nominalisation or reification. Conversely, from a singular term for a property one can obtain a predicate that expresses the property, e.g., 'the property $\Phi$ (qua object) applies to ...'. For to say that the property $\Phi$ applies to the $X \mathrm{~s}$ is equivalent to predicating $\Phi$ of the $X \mathrm{~s}$.

The functor $\lambda$ has been introduced by stipulation; its role is to change a predicate expressing a property into a singular term for that property. The $\lambda$-operation is defined by this stipulation. The content of the definition, namely that what $\lambda_{X} \Phi(X)$ refers to is the same as what $\Phi$ expresses, cannot be fully

\footnotetext{
${ }^{4}$ Note that the modality $\square$ operates on properties, not propositions; it signals necessary satisfaction, not necessary truth.
} 
represented in the formal language at our disposal. Any attempt to formulate the sameness in question will be ungrammatical. Hence, the formula (2.2) is not a definition, but it records the effect of the stipulation to the extent that it can be expressed in the symbolism. I therefore call the formula a postulate. In (2.2) the symbol $\lambda$ is not uninterpreted and in contrast with the functor 'the number of' in Hume's Principle there is no room for alternative interpretations.

Being a purely syntactic device the $\lambda$-operator is of no metaphysical significance. Even though the property is treated as an object, its primary category remains that of a property. Semantic categories are best identified syntactically. So the primary semantic category of a property is identified with the syntactic category of a predicate expressing it. Qua object, the (secondary) category of the property is the category of a singular term. All items that can be referred to by singular terms will be called objects. Individuals are those objects whose primary category is the category of objects. ${ }^{5}$ Objects whose primary category is not the category of objects are abstract objects.

The legitimacy of treating properties as objects turns on the point that the truth-conditions of all propositions about these objects are well defined. For every property $\mathcal{F}$ of properties of pluralities there is a property $\varphi$ of their reifications:

$$
\varphi\left(\lambda_{X} \Psi(X)\right) \equiv \mathcal{F}_{X} \Psi(X) .
$$

Therefore any claim that can be made about a property when expressed by a predicate can also be made when the property is treated as an object. This goes specifically for numerical properties. All properties of numbers are obtained from properties of numerical properties. Every predicate that expresses a property of numbers is a grammatical modification of a predicate of numerical properties. $^{6}$

\footnotetext{
${ }^{5}$ This explanation of the role of the $\lambda$-functor is closely related to an account suggested in [Hale and Wright, 2012]. A distinction is drawn there between primary and secondary modes of reference. In the case of a property the primary mode of reference is a predicate, say 'is a planet', whereas the singular term 'the property of being a planet' functions as a secondary mode of reference. Seeing that not only singular terms may refer to properties, but that a property may also be one of the referents of a plural term and therefore be in the range of a first-order quantifier, I prefer to say that the property itself belongs to different semantic categories. Its primary category is the category of properties of pluralities; its secondary category is the category of objects.

${ }^{6}$ The possibility of representing items in some category by objects is not restricted to properties. Whenever there exists for a semantic category an analogue of identity it is possible to introduce abstract objects whose identity is given by that analogue of identity. Abstract objects representing items in this category are different from those representing other categories; in particular, they are different from individuals. Abstract objects do not form just one undivided category; they differ from one another depending on what their primary categories are.
} 


\subsection{Logical Properties}

The position we have arrived at is that numbers are properties of pluralities, treated as objects. What needs to be done next is to select from among all the properties of pluralities those that are numbers, the numerical properties. Numerical properties are the ones that satisfy Hume's Principle.

To begin with we note that numerical properties are non-contingent. The relation of equinumerosity between pluralities is non-contingent. If the elements of two pluralities can be paired with one another then this is necessarily so; if there is no such pairing, then it is impossible to match them one-to-one. Therefore the properties that are associated with the equivalence relation of equinumerosity are also non-contingent. If such a property $\Phi$ is true of certain $X \mathrm{~s}$, then $\Phi$ applies to the $X \mathrm{~s}$ necessarily. The necessity here is de re necessity (or perhaps de rebus necessity). Non-contingent properties can be characterised as follows.

$$
(\forall X)[\square \Phi(X) \vee \square \sim \Phi(X)] .
$$

The non-contingent properties are the logical properties, i.e., those that invoke only logical notions, including identity, and can be expressed using only logical vocabulary. A further valuable feature of these properties is that they are applicable to any objects, irrespective of their primary categories; logical properties are syncategorematic, in the sense that the application conditions of a logical predicate are determinate for objects of any category. Conversely, any predicate that can meaningfully be applied to any object or objects must be logical.

Drawing attention to the fact that the numerical properties are logical properties serves to clarify the sense in which numbers are, as Frege maintains, logical objects. Numbers are reifications of certain logical properties of pluralities, properties that apply to a plurality necessarily if they apply at all to that plurality.

\subsection{Numerical Properties}

Not all logical objects are numbers. Numbers need to be identified by what distinguishes them from other logical objects. Hume's Principle tells us that when a number applies to a plurality it applies to another plurality if and only if the two pluralities are equinumerous. This means that numerical properties are strictly congruent. ${ }^{7}$

$$
(\forall X)(\forall Y) \square[\Phi(X) \supset(X \approx Y \equiv \Phi(Y))] .
$$

However, (2.4) is not sufficient. For the impossible property is also strictly congruent. It has to be ruled out; for every number it has to be at least possible

\footnotetext{
${ }^{7}$ Since equinumerosity is a collective relation between pluralities, the numerical properties themselves are collective properties.
} 
that there is a plurality which it applies to.

$$
\diamond(\exists X) \Phi(X) .
$$

Numerical properties are the properties of pluralities that satisfy (2.3), (2.4), and (2.5); they are the logical properties that are strictly congruent with respect to equinumerosity and consistent. Not surprisingly, it turns out that the numerical quantifiers, such as 'there are two ...', express numerical properties. ${ }^{8,9}$ Nominalisation applied to numerical properties delivers the cardinal numbers and the cardinal numbers are the only abstract objects we are interested in.

The nominalisation functor $\lambda$ is applicable to $\Phi$ iff $\Phi$ is a numerical property.

We are now in possession of a definition of the cardinal numbers in two parts: (2.2) characterises the functor $\lambda ;(2.6)$ specifies its domain of application.

\subsection{Determinate Pluralities}

The numerical properties qua objects are numbers, more precisely the cardinal numbers. When $\Phi$ is a numerical property then $\lambda_{X} \Phi(X)$ is a cardinal number. With the cardinal numbers on hand we can now turn to the functor 'the (cardinal) number of'. Introducing the functor is justified if every plurality has just one numerical property. That for every plurality there is at most one numerical property is obvious. For (2.4) guarantees that if two numerical properties apply to the same plurality they are necessarily coextensive and hence are identical qua cardinal numbers.

But we have no reason to assume that every plurality has a cardinal number. Some collective properties, in particular number properties, can only be applied to a plurality if it is determinate what the elements of the plurality are. One cannot attribute a number to the plurality of all sets or the plurality of all objects, because the application of number predicates requires that the plurality in question has determinate boundaries; it has to be determinate which elements belong to it. ${ }^{10}$ Therefore the functor 'the number of' can be applied

\footnotetext{
${ }^{8}$ ' $\left(\exists_{n} y\right) y \in \in X$ ' expresses a numerical property of pluralities. The formula will be abbreviated to ' $\exists \exists_{n}(X)$ '.

${ }^{9}$ There is a significant affinity with David Bostock's account [1980] of the natural numbers as type-neutral numerical quantifiers. While the present account is concerned with first-order numerical quantifiers ' $\left(\exists_{n} x\right) \ldots x \ldots$ ' with the variable $x$ ranging over objects, the variable $\xi$ in Bostock's quantifiers ' $\left(\exists_{n} \xi\right) \ldots \xi \ldots$ ' is a placeholder for the variables corresponding to all types in the logical hierarchy.

${ }^{10}$ In the cases of interest to us the indeterminacy can be attributed to the infinite extensibility of the concept set or object. In other cases the vagueness of a plural term is to blame. It is noteworthy that even when the indeterminacy of a plurality means that certain collective predicates cannot be applied, distributive predicates are not necessarily affected. A first-level universal or existential quantifier whose range is an indeterminate plurality is still significant. It may, e.g., be possible to ascertain that every element of such a plurality has a certain property on the basis of the sense of a plural term that refers to the plurality.
} 
only to determinate pluralities. Reference to indeterminate pluralities has to be avoided and the range of plural quantifiers has to be restricted to determinate pluralities. However, this restriction cannot be made explicit, since there is no formal characterisation of determinate pluralities available. In the absence of such a characterisation restricting the range of plural quantifiers to determinate pluralities consists in ensuring that the theory does not allow the formation of any plural term that refers to an indeterminate plurality.

With the restriction to determinate pluralities in place we can regard the functor 'the number of' as total. Every determinate plurality, the $X \mathrm{~s}$, has a unique numerical property, hence a unique number, namely the reification of the property that any $Y \mathrm{~s}$ have if, and only if, they are equinumerous with the $X \mathrm{~s}$, irrespective of how they are referred to, and so Hume's Principle is secured.

\subsection{Properties of Numbers}

In order to formulate statements about numbers there need to be predicates for them. Predicates of individuals other than logical ones will not be significant when applied to numbers. What we are interested in are mathematical properties such as the property of being a prime number. The relevant properties are delivered automatically thanks to the presence of the $\lambda$-functor. For as numbers come from the numerical properties of pluralities, so the properties of numbers come from the properties of the numerical properties of pluralities, and these in turn correspond to congruence properties of pluralities.

Let $F$ be a property of numerical properties of pluralities. For fixed cardinal number $x, x=\operatorname{nr}(Z)$ is a numerical property of pluralities. Hence $F_{Z}(x=$ $n r(Z))$ can be used to define a property $\varphi$ of numbers.

$$
\varphi(x) \equiv F_{Z}(x=n r(Z)) .
$$

From $\varphi$ one obtains a property $\Phi$ of pluralities, the property that the $X \mathrm{~s}$ have if the number of $X \mathrm{~s}$ has property $\varphi$.

$$
\Phi(X) \equiv \varphi(n r(X)) .
$$

Expressed in terms of $F$,

$$
\Phi(X) \equiv F_{Z}(n r(X)=n r(Z)),
$$

i.e.,

$$
\Phi(X) \equiv F_{Z}(X \approx Z) .
$$

$\Phi$ need not be a numerical property, but it is a congruence property. For assume $X \approx Y$. Then

$$
F_{Z}(X \approx Z) \equiv F_{Z}(Y \approx Z)
$$

and so

$$
\Phi(X) \equiv \Phi(Y)
$$

Therefore $\Phi$ satisfies

$$
(\forall X)(\forall Y)[X \approx Y \supset(\Phi(X) \equiv \Phi(Y))] .
$$


Hence if $\varphi$ is a property of numbers then $\Phi(X)$, defined as $\varphi(n r(X))$, is a congruence property under equinumerosity. Conversely, from any congruence property $\Phi(X)$ one obtains a property $\varphi$ of numbers by stipulating

$$
\varphi(x) \equiv(\forall Y)(x=n r(Y) \supset \Phi(Y)) .
$$

Analogous considerations show that the relations between numbers correspond to the congruence relations between pluralities. This result is not a new discovery. We have already seen that any category of objects that satisfy Hume's Principle has the congruence properties as its characteristic properties.

Turning to the properties of pluralities of numbers, the ones we need for mathematics are just the logical properties. The application of numerical predicates to pluralities consisting of numbers is therefore unproblematic. Propositions such as 'there are four prime numbers between 10 and 20' have determinate truth-conditions, since the logical predicate 'there are four $X \mathrm{~s}$ ' has a definite meaning which is unaffected by the enlargement of its range of applicability to include numbers.

With numbers and their properties at hand arithmetic can be developed. The cardinal numbers and then the natural numbers need to be formally introduced. When the basic arithmetical notions are defined, arithmetical statements will have been given a meaning in terms of logical concepts, and the true arithmetical statements emerge as logical truths.

\section{THE DEFINITION OF NUMBERS}

This section gives a sketch of the formal development of the theory of natural numbers from definitions. Cardinal numbers and their basic properties are introduced. The natural numbers are defined in the familiar recursive way. Finally it is shown that the logic is strong enough to derive the counterparts of the second-order Peano Axioms.

\subsection{The Language of Arithmetic}

As the discussion so far has shown, the language in which arithmetic is developed has to be many-sorted and modal, and it has to allow for both plural quantification (extensional) and second-order quantification (intensional). As we now turn to the task of showing how the definitions employed lead to the truth-conditions of the statements of arithmetic, it is advisable to reduce the language to the minimum that is needed for this task. This means that all nonlogical vocabulary is excluded. The resulting language $L$ can do without modal operators, since all its predicates are constructed from logical vocabulary and are therefore non-contingent.

$L$ features singular and plural terms, singular and plural predicates and relations, singular and plural quantification, as well as quantifiers ranging over singular and plural properties. Moreover, the definition of equinumerosity involves quantifying over functions, which are understood extensionally. The variables for objects are $x, y, \ldots$; the variables $x^{c}, y^{c}, \ldots$ range over numbers, a 
sub-category of objects. The letters $\varphi$ and $\psi$ are used as variables for predicates that are applicable to numbers.

The grammar of plural logic is similar to that of sets. ${ }^{11}$ The plural variables $X, Y, \ldots$ range over determinate pluralities of objects, including the empty plurality and one-element pluralities. Since only determinate pluralities are recognised in the theory it is a requirement that the referents of any plural term, in particular of any plural constant, are a determinate plurality. When a plurality is not determinate it is not represented by a plural term but by singular variables that range over that plurality. Capital Greek letters $\Phi, \Psi, \ldots$ are used as variables for predicates of pluralities.

There are complex terms of the form $\langle x \in \in F \mid \varphi(x)\rangle$, which refer to all those elements of the plurality of $F$ s that have the property $\varphi$. If the $F$ s are a determinate plurality, as is now assumed, and $\varphi$ has definite application conditions, then $\langle x \in \in F \mid \varphi(x)\rangle$, the $F$ s that are $\varphi$, are also a determinate plurality. ${ }^{12}$ When a plurality is not determinate, and is represented by, say, the variable $v$ rather than by a plural term, then the plural term $\langle v \mid \varphi(v)\rangle$ refers to those objects in the range of $v$ that have property $\varphi$, provided that these objects are a determinate plurality. Otherwise new singular variables have to be introduced to range over the $v$-objects that are $\varphi$.

As to relations among pluralities, $X==Y$ signifies that the $X$ s are identical with the $Y \mathrm{~s}, x \in \in Y$ symbolises ' $x$ is one of the $Y \mathrm{~s}$ ', $X \subseteq \subseteq Y$ expresses 'the $X \mathrm{~s}$ are among the $Y \mathrm{~s}$ ', and $X \approx Y$ will be defined to mean that there are as many $X \mathrm{~s}$ as there are $Y \mathrm{~s}$.

Restricted quantification can be expressed with the help of plural terms. ' $(\forall x \in \in F) \ldots x \ldots$. abbreviates ' $(\forall x)(x \in \in F \supset \ldots x \ldots)$ ' and ' $(\forall X \subseteq \subseteq$ $F) \ldots X \ldots$. abbreviates ' $(\forall X)(X \subseteq \subseteq F \supset \ldots X \ldots)$ '.

\subsection{Cardinal Numbers}

\subsubsection{Equinumerosity}

Two pluralities are equinumerous if there is a one-one function that maps the elements of one onto the elements of the other.

$$
\begin{gathered}
X \approx Y \equiv(\exists f)[(\forall x \in \in X) f(x) \in \in Y \\
\&(\forall y \in \in Y)(\exists x \in \in X)(\forall z \in \in X)(f(z)=y \equiv z=x)] .
\end{gathered}
$$

\subsubsection{Nominalisation}

The $\lambda$-operator turns predicates of pluralities into singular terms. The abstract objects so introduced are identical if the properties in question necessarily have the same instances. But since $L$ has only logical vocabulary, this means no more

\footnotetext{
${ }^{11}$ The symbolism used here is largely borrowed from [Burgess, 2004]. A detailed account of plural quantification is given by Linnebo [2004].

${ }^{12}$ The referents of $\langle x \mid \varphi(x)\rangle$ are the objects that a comprehension principle, usually part of plural logic, would associate with the property $\varphi$.
} 
than that the properties are coextensive.

$$
\left(\lambda_{X} \Phi(X)=\lambda_{X} \Psi(X)\right) \equiv(\forall X)(\Phi(X) \equiv \Psi(X))
$$

The intended domain of application of the $\lambda$-functor are the numerical properties. A numerical property is a logical property that is strictly congruent and which can be instantiated. Since the only properties recognised in $L$ are logical ones, only strict congruence and instantiability need to be mentioned in the definition. In the non-modal language $L$ the condition that property $\Phi$ is strictly congruent is

$$
(\forall X)(\forall Y)[\Phi(X) \supset(X \approx Y \equiv \Phi(Y))]
$$

And the requirement that any numerical property can have instances becomes the requirement that any such property does have instances:

$$
(\exists X) \Phi(X) .
$$

So, using $\mathcal{N}$ for 'numerical property' we have

$$
\mathcal{N}_{X} \Phi(X) \equiv\{(\forall X)(\forall Y)[\Phi(X) \supset(X \approx Y \equiv \Phi(Y))] \&(\exists X) \Phi(X)\}
$$

The desired nominalisation principle for numerical properties now is

$$
\begin{gathered}
\left(\mathcal{N}_{X} \Phi(X) \& \mathcal{N}_{X} \Psi(X)\right) \supset \\
{\left[\left(\lambda_{X} \Phi(X)=\lambda_{X} \Psi(X)\right) \equiv(\forall X)(\Phi(X) \equiv \Psi(X))\right] .}
\end{gathered}
$$

\subsubsection{Cardinal Numbers}

Seeing that the plurality of cardinal numbers is indeterminate, the cardinal numbers are introduced, not as the referents of a plural term, but as the range of quantification indicated by the number variables $x^{c}, y^{c}, \ldots$. Every cardinal number is the reification of a numerical property of pluralities and the reification of every numerical property is a cardinal number.

$$
\begin{aligned}
& (\forall \Phi)\left[\mathcal{N}_{X} \Phi(X) \supset\left(\exists x^{c}\right) x^{c}=\lambda_{Y} \Phi(Y)\right] ; \\
& \left(\forall x^{c}\right)(\exists \Phi)\left[\mathcal{N}_{X} \Phi(X) \& x^{c}=\lambda_{Y} \Phi(Y)\right] .
\end{aligned}
$$

Finally, cardinal numbers are objects.

$$
\left(\forall x^{c}\right)(\exists y) x^{c}=y
$$

The postulates (N1)-(N3) give expression to the stipulation that, as regards any numerical property $\Phi$, the singular term $\lambda_{X} \Phi(X)$ refers to the property as an object. 
The application relation @, which relates numbers to pluralities, can now be defined. $x^{c} @ Y$ says that the number $x^{c}$ applies to the plurality $Y$.

$$
x^{c} @ Y \equiv(\exists \Phi)\left(\mathcal{N}_{Z} \Phi(Z) \& x^{c}=\lambda_{Z} \Phi(Z) \& \Phi(Y)\right) .
$$

The effect of @ is to reverse the $\lambda$-operation; for a given number $x^{c}, x^{c} @ Y$ is the numerical property of which $x^{c}$ is the reification.

$$
\begin{gathered}
\left(\forall x^{c}\right) \mathcal{N}_{Y}\left(x^{c} @ Y\right) ; \\
\left(\forall x^{c}\right)\left(x^{c}=\lambda_{Y}\left(x^{c} @ Y\right)\right) .
\end{gathered}
$$

Two further consequences of the definition are these:

$$
\begin{gathered}
\left(\forall x^{c}\right)(\exists Y) x^{c} @ Y ; \\
\left(\forall x^{c}\right)\left(\forall y^{c}\right)\left[(\forall Z)\left(x^{c} @ Z \equiv y^{c} @ Z\right) \supset x^{c}=y^{c}\right] . \\
\text { 3.2.4. The functor 'the number of' }
\end{gathered}
$$

If the $X \mathrm{~s}$ are a determinate plurality, as we assume, then there is a unique numerical property and hence a unique cardinal number that applies to them, and so the functor 'the number of' can be introduced.

First note that if a cardinal number applies to a plurality then it is unique. For assume that both $x^{c} @ W$ and $y^{c} @ W$. Then $(\forall Z)\left(Z \approx W \equiv x^{c} @ Z\right)$ and $(\forall Z)\left(Z \approx W \equiv y^{c} @ Z\right)$ by (3.1) and (D2). Hence $(\forall Z)\left(x^{c} @ Z \equiv y^{c} @ Z\right)$, i.e., $x^{c}=y^{c}$ by (3.4). So, at most one number applies to any plurality.

$$
\left(\forall x^{c}\right)\left(\forall y^{c}\right)(\forall Z)\left[\left(x^{c} @ Z \& y^{c} @ Z\right) \supset x^{c}=y^{c}\right] .
$$

Also, given a determinate plurality, there is at least one number that applies to it. For one can identify a numerical property that the elements of the plurality have. The property that the $Y \mathrm{~s}$ have if they are equinumerous with the $X \mathrm{~s}$ is a numerical property that the $X$ s themselves have. Therefore its reification is a number that applies to the $X$ s. So we adopt the following definition. ${ }^{13}$

$$
n r(X)=\lambda_{Y}(Y \approx X)
$$

It is immediately obvious that Hume's Principle,

$$
(\forall X)(\forall Y)(n r(X)=n r(Y) \equiv X \approx Y),
$$

is satisfied if the functor 'the number of' is defined by (D4). (D3) and (D4) also imply

$$
\left(\forall x^{c}\right)(\forall Y)\left(x^{c}=n r(Y) \equiv x^{c} @ Y\right)
$$

and consequently

$$
(\forall X)(n r(X) @ X) .
$$

\footnotetext{
${ }^{13}$ It has to be noted that this simple definition is available only because $L$ does not allow for non-logical vocabulary.
} 


\subsection{Natural Numbers}

The aim of this section is to show that the propositions of arithmetic can be expressed in $L$ and that, thanks to the postulates (N1)-(N3) and definitions (D1)-(D4), they have the right truth-conditions. The natural numbers are the reifications of the finite numerical properties, i.e., the numerical quantifiers $\exists_{n}(X)$. The sequence of numerical quantifiers is given by (3.8).

$$
\begin{gathered}
\exists_{0}(X) \equiv \sim(\exists y) y \in \in X \\
\exists_{n+1}(X) \equiv(\exists y \in \in X) \exists_{n}(\langle w \in \in X \mid w \neq y\rangle) .
\end{gathered}
$$

The number $n$ is the reification of the numerical quantifier $\exists_{n}$; so the individual natural numbers are introduced by infinitely many definitions of the form

$$
n=\lambda_{X} \exists_{n}(X) .
$$

The plurality of natural numbers will be introduced, in the usual way, by means of an inductive definition with the help of the successor relation. The definition of the successor relation invokes the congruence relation 'there are at least as many $Y \mathrm{~s}$ as there are $X \mathrm{~s}$ ', from which the ordering relations $x^{c} \leq y^{c}$ and $x^{c}<y^{c}$ among cardinal numbers are obtained.

$$
\begin{gathered}
X \precsim Y \equiv(\exists Z \subseteq \subseteq Y) X \approx Z ; \\
x^{c} \leq y^{c} \equiv(\forall X)(\forall Y)\left[\left(x^{c} @ X \& y^{c} @ Y\right) \supset X \precsim Y\right] ;^{14} \\
x^{c}<y^{c} \equiv x^{c} \leq y^{c} \& x^{c} \neq y^{c} .
\end{gathered}
$$

The successor relation is defined on all numbers as follows.

$$
\sigma\left(x^{c}, y^{c}\right) \equiv(\forall Y)\left[y^{c} @ Y \equiv(\exists u \in \in Y) x^{c} @\langle w \in \in Y \mid w \neq u\rangle\right] .
$$

Some immediate consequences of the definitions are the following.

$$
\begin{gathered}
\sim\left(\exists x^{c}\right) x^{c}<0 ; \\
\sim\left(\exists x^{c}\right) \sigma\left(x^{c}, 0\right) ; \\
\left(\forall x^{c}\right)\left(\forall y^{c}\right)\left(\sigma\left(x^{c}, y^{c}\right) \supset x^{c} \leq y^{c}\right) ; \\
\left(\forall x^{c}\right)\left(\forall y^{c}\right)\left(\forall z^{c}\right)\left[\left(\sigma\left(x^{c}, y^{c}\right) \& \sigma\left(x^{c}, z^{c}\right)\right) \supset y^{c}=z^{c}\right] ; \\
\left(\forall x^{c}\right)\left(\forall y^{c}\right)\left(\forall z^{c}\right)\left[\left(\sigma\left(x^{c}, z^{c}\right) \& \sigma\left(y^{c}, z^{c}\right)\right) \supset x^{c}=y^{c}\right] .
\end{gathered}
$$

(D9) does not imply that every number has a successor, nor that a successor is always distinct from the preceding number. However, once the natural numbers are defined it can be proved that every natural number has a distinct successor.

\footnotetext{
${ }^{14}$ (D7) is not sufficient to establish that the cardinals are totally ordered. $\left(\forall x^{c}\right)\left(\forall y^{c}\right)\left(x^{c} \leq y^{c} \vee y^{c} \leq x^{c}\right)$ is guaranteed only if a version of the axiom of choice is added to plural logic.
} 
Since the natural numbers are a determinate plurality, they are introduced, in the familiar recursive way, as the referents of the plural term NN.

$$
\begin{gathered}
x^{c} \in \in \mathrm{NN} \equiv \\
(\forall X)\left\{\left[0 \in \in X \&\left(\forall y^{c} \in \in X\right)\left(\forall z^{c}\right)\left(\sigma\left(y^{c}, z^{c}\right) \supset z^{c} \in \in X\right)\right] \supset x^{c} \in \in X\right\} .
\end{gathered}
$$

From this we obtain immediately the Principle of Induction in the plurality form

$$
(\forall X)\left\{\left(0 \in \in X \&\left(\forall y^{c} \in \in X\right)\left(\forall z^{c}\right)\left(\sigma\left(y^{c}, z^{c}\right) \supset z^{c} \in \in X\right) \supset \mathrm{NN} \subseteq \subseteq X\right\}\right.
$$

and in the more familiar property form

$$
\begin{aligned}
(\forall \varphi)\left\{\left(\varphi ( 0 ) \& \left(\forall y^{c}\right.\right.\right. & \in \in \mathrm{NN})\left(\forall z^{c}\right)\left(\sigma\left(y^{c}, z^{c}\right) \supset\left(\varphi\left(y^{c}\right) \supset \varphi\left(z^{c}\right)\right)\right) \\
& \left.\supset\left(\forall x^{c} \in \in \mathrm{NN}\right) \varphi\left(x^{c}\right)\right\} .
\end{aligned}
$$

That the successor of a number, as defined by (D9), is different from that number is true of the natural numbers, but not of the larger cardinals. To establish the result that $\left(\forall x^{c} \in \in \mathrm{NN}\right)\left(\forall y^{c} \in \in \mathrm{NN}\right)\left(\sigma\left(x^{c}, y^{c}\right) \supset x^{c}<y^{c}\right)$, inductive reasoning, as formulated in (3.15), is required. Clearly, if $\sigma\left(0, y^{c}\right)$, then $y^{c} \neq 0$, and hence $0<y^{c}$ by (3.11). Now assume that $x^{c} \in \in \mathrm{NN}, \sigma\left(x^{c}, y^{c}\right)$ and $\sigma\left(y^{c}, z^{c}\right)$. $x^{c}<y^{c}$ by the inductive hypothesis. Suppose for reductio that $y^{c}=z^{c}$. Then $(\forall Z)\left[y^{c} @ Z \equiv(\exists u \in \in Z) x^{c} @\langle w \in \in Z \mid w \neq u\rangle\right]$, since $\sigma\left(x^{c}, y^{c}\right) ; \quad(\forall Z)\left[z^{c} @ Z \equiv\right.$ $\left.(\exists u \in \in Z) y^{c} @\langle w \in \in Z \mid w \neq u\rangle\right]$, since $\sigma\left(y^{c}, z^{c}\right)$; and $(\forall Z)\left(y^{c} @ Z \equiv z^{c} @ Z\right)$, since $y^{c}=z^{c}$. Therefore $(\forall Z)\left[(\exists u \in \in Z) x^{c} @\langle w \in \in Z \mid w \neq u\rangle \equiv(\exists u \in \in Z) y^{c} @\langle w \in \in\right.$ $Z|w \neq u\rangle]$, and hence $x^{c}=y^{c}$, q.e.a. Therefore $y^{c} \neq z^{c}$ and so $y^{c}<z^{c}$. So

$$
\left(\forall x^{c} \in \in \mathrm{NN}\right)\left(\forall y^{c} \in \in \mathrm{NN}\right)\left(\sigma\left(x^{c}, y^{c}\right) \supset x^{c}<y^{c}\right) .
$$

It follows that if $y^{c}$ succeeds $x^{c}$, then there is no number between $x^{c}$ and $y^{c}$.

$$
\left(\forall x^{c} \in \in \mathrm{NN}\right)\left(\forall y^{c} \in \in \mathrm{NN}\right)\left[\sigma\left(x^{c}, y^{c}\right) \supset\left(\forall w^{c}\right)\left(w^{c} \leq x^{c} \equiv w^{c}<y^{c}\right)\right] .
$$

A further inductive argument establishes that $\left(\forall x^{c} \in \in \mathrm{NN}\right) x^{c} @\left\langle w^{c}\right| w^{c}<$ $\left.x^{c}\right\rangle$. Clearly, 0@ $\left\langle w^{c} \mid w^{c}<0\right\rangle$. Now assume $x^{c} \in \in \mathrm{NN}, \sigma\left(x^{c}, y^{c}\right)$, and for induction $x^{c} @\left\langle w^{c} \mid w^{c}<x^{c}\right\rangle$. Then $x^{c}<y^{c}$ by (3.16) and $y^{c} @\left\langle w^{c} \mid w^{c} \leq x^{c}\right\rangle$ by the definition of $\sigma$. Hence $y^{c} @\left\langle w^{c} \mid w^{c}<y^{c}\right\rangle$ by (3.17). Therefore

$$
\left(\forall x^{c} \in \in \mathrm{NN}\right) x^{c} @\left\langle w^{c} \mid w^{c}<x^{c}\right\rangle
$$

and, equivalently,

$$
\left(\forall x^{c} \in \in \mathrm{NN}\right) x^{c}=n r\left(\left\langle w^{c} \mid w^{c}<x^{c}\right\rangle\right) .
$$

Finally, every natural number $x^{c}$ has $n r\left(\left\langle w^{c} \mid w^{c} \leq x^{c}\right\rangle\right)$ as its successor. For $x^{c} @\left\langle w^{c} \mid w^{c}<x^{c}\right\rangle$ by (3.18), i.e., $(\exists u) x^{c} @\left\langle w^{c} \mid w^{c} \leq x^{c} \& w^{c} \neq u\right\rangle$. And besides 


$$
\begin{gathered}
n r\left(\left\langle w^{c} \mid w^{c} \leq x^{c}\right\rangle\right) @\left\langle w^{c} \mid w^{c} \leq x^{c}\right\rangle \text { by }(3.7) \text {. Hence } \sigma\left(x^{c}, n r\left(\left\langle w^{c} \mid w^{c} \leq x^{c}\right\rangle\right)\right. \text {. So, } \\
\left(\forall x^{c} \in \in \mathrm{NN}\right) \sigma\left(x^{c}, n r\left(\left\langle w^{c} \mid w^{c} \leq x^{c}\right\rangle\right)\right)
\end{gathered}
$$

which means that every natural number has a successor:

$$
\left(\forall x^{c} \in \in \mathrm{NN}\right)\left(\exists y^{c} \in \in \mathrm{NN}\right) \sigma\left(x^{c}, y^{c}\right) .
$$

This completes the explication of basic arithmetical notions in terms of numerical properties of pluralities and their properties. The plurality of natural numbers has been introduced as well as the ordering relation $<$ and the successor relation $\sigma$. It has been shown that the Induction Principle holds and that the successor relation has the right properties. This is sufficient to justify the Peano Axioms. Other arithmetical notions, like addition and multiplication, can be defined in ways that are analogous to the definitions that are familiar from second-order arithmetic. The definitions provide for the truth-conditions of arithmetical statements.

\section{CONCLUSION}

The account of the arithmetic of natural numbers in this paper vindicates not only Frege's logicist thesis, but also to a significant extent his strategy of identifying the natural numbers with certain logical objects which satisfy Hume's Principle. The details of the present account are of course different. The (cardinal) numbers have been identified not as sets, but as certain properties, namely the numerical properties of pluralities, these properties being treated as objects.

The acceptability of the account might be queried in two ways. It may seem doubtful whether it is legitimate to introduce objects by definition and whether the principles employed in drawing inferences from definitions can be called logical in a sense that is close enough to Frege's intentions.

The logic employed certainly goes beyond what Frege recognised as logic. The logic of plurals is a recent development which replicates some of the descriptive capacity of extensional second-order logic and so does not really add to the strength of Frege's logical system. But the intensional treatment of predicates is something that Frege did not envisage. It is an important aspect of the present approach, because it leaves the range of applicability of a predicate open and it separates the nominalisation operation from the extension of the predicate it operates on, thereby ensuring that no contingent matters affect arithmetic. Since the modal notion invoked in the identity condition of properties is logical necessity, a notion which is central to Frege's conception of logicism, there is no good reason to object to its playing a role in the object language.

The introduction of numbers as objects is basically no more than a grammatical transformation which makes it possible to refer to numbers by singular and plural terms. The notion of object invoked here is admittedly a weak one. However, any more substantial notion is irrelevant, since the judgment that in mathematical practice numbers are objects is itself based solely on grammatical grounds. The introduction of singular and plural terms for numbers is accompanied by the specification of a range of properties of numbers. As a result of 
the stipulation number-theoretic statements are provided with truth-conditions. This is sufficient reason to regard the stipulations as definitions.

Hence I see no compelling reason to deny that the present account of the natural numbers can be regarded as a logicist account.

\section{REFERENCES}

Bostock, David [1980]: 'A study of type-neutrality', Journal of Philosophical Logic 9, 211-296, 363-414.

Burgess, John P. [2004]: 'E pluribus unum: Plural logic and set theory', Philosophia Mathematica (3) 12, 193-221.

Dummett, Michael [1998]: 'Neo-Fregeans: In bad company?', in M. Schirn, ed, Philosophy of Mathematics Today, pp. 368-388. Oxford: Clarendon Press.

Frege, Gottlob [1893]: Grundgesetze der Arithmetik. I. Band. Hildesheim: Georg Olms. Reprinted 1961.

Hale, Bob, and Crispin Wright [2001]: The Reason's Proper Study: Essays Towards a Neo-Fregean Philosophy of Mathematics. Oxford: Clarendon Press.

[2012]: 'Horse sense', The Journal of Philosophy 109, 85-131.

Heck, Richard G., Jr [1993]: 'The development of arithmetic in Frege's Grundgesetze der Arithmetik', Journal of Symbolic Logic 58, 579-601.

Linnebo, Øystein [2004]: 'Plural quantification', The Stanford Encyclopedia of Philosophy (Spring 2013 Edition), E.N. Zalta, ed. (online): http://plato.stanford.edu/ archives/spr2013/entries/plural-quant/.

Tennant, Neil [2014]: 'Logicism and neologicism', The Stanford Encyclopedia of Philosophy (Spring 2014 Edition), E.N. Zalta, ed. (online): http://plato.stanford.edu/ archives/fall2014/entries/logicism/.

Trueman, Robert [2014]: 'A dilemma for neo-Fregeanism', Philosophia Mathematica (3) 22, 361-379. 